\title{
Balkanologie
}

Balkanologie Revue d'études pluridisciplinaires

Vol. X, $n^{\circ} 1-2 \mid 2008$

Volume $X$ Numéro 1-2

\section{La coopération internationale sur le Danube : Géopolitique de l'intégration du fleuve au continent européen}

Jean-Marie Gauthey

\section{CpenEdition}

Journals

Édition électronique

URL : https://journals.openedition.org/balkanologie/409

DOI : 10.4000/balkanologie.409

ISSN : 1965-0582

Éditeur

Association française d'études sur les Balkans (Afebalk)

Référence électronique

Jean-Marie Gauthey, « La coopération internationale sur le Danube : Géopolitique de l'intégration du fleuve au continent européen », Balkanologie [En ligne], Vol. X, n 1-2 | 2008, mis en ligne le 09 juin 2008, consulté le 28 juin 2022. URL : http://journals.openedition.org/balkanologie/409 ; DOI : https:// doi.org/10.4000/balkanologie.409

Ce document a été généré automatiquement le 17 décembre 2020.

(c) Tous droits réservés 


\title{
La coopération internationale sur le Danube : Géopolitique de l'intégration du fleuve au continent européen
}

\author{
Jean-Marie Gauthey
}

«L'histoire danubienne est riche de projets jamais réalisés- de fédérations plurinationales, depuis la confédération germano-magyare slavo-

latine ou la république fédérale du Danube ouverte à toutes les nationalités -conçues par le baron Miklos Wessélenyi respectivement en 1842

et 1849- jusqu'au programme multinational

d'Istvan Széchényi en 1849, depuis le tardif repentir de Kossuth [...] jusqu'au grandiose projet du Roumain Aurel Popovici, intitulé "Les ÉtatsUnis de la Grande Autriche". Aucun de ces projets

n'a jamais connu l'ombre d'une réalisation »

(Claudio Magris, Danube, 1988)' ${ }^{1}$.

1 Les grands fleuves constituent un élément physique communément considéré comme façonnant l'espace. Le Danube dispose à ce titre d'une position toute particulière compte tenu de ses dimensions et de son orientation est-ouest atypique en Europe qui lui font traverser successivement dix pays différents. Ce fleuve crée un dénominateur commun entre ses peuples riverains. Il a assumé « au cours de l'histoire des fonctions complexes et parfois contradictoires, où reviennent sans cesse les problèmes d'unité recherchée et d'espace contesté ou partagé2».

2 Il serait toutefois abusif de concevoir que les transformations politiques dans le bassin du Danube révolutionnent son ordonnancement. Ces mutations, certes profondes, se composent d'éléments de permanence hérités des systèmes antérieurs et de leur 
inertie. Même les dynamiques novatrices et originales relèvent traditionnellement d'impulsions extérieures au bassin suivant l'influence historique des puissances européennes dans la région. Le statut du fleuve, et par là les questions de la gestion et de l'organisation politique de son bassin, permet d'envisager les changements que connaît la région et de proposer des modes de résolution qui s'intègrent à la dynamique de l'Union européenne (UE). L'étude de ce processus en cours passe par la décomposition analytique $\mathrm{du}$ jeu des forces en présence, des acteurs, de leurs interactions et des composantes structurelles et conjoncturelles dans leur cadre géographique. Cette déconstruction permet de révéler les enjeux actuels de la recomposition géopolitique du bassin danubien.

3 Le Danube apparaît comme un fleuve international dissocié en sous-systèmes plus ou moins imbriqués et réellement autonomes. La réflexion sur l'intégration politique de son bassin s'appuie bien sur certaines données tangibles et des arguments relativement objectifs mais elle souffre de présupposés idéologiques tenaces qui poussent ses habitants à la différenciation et parfois à la mésentente.

4 Les formes de coopération politique autour du fleuve sont aussi nombreuses qu'incomplètes. L'avenir du Danube semble à présent se lire dans l'Union européenne. Mais il traverse toute une série d'imbroglios géopolitiques, essentiellement sur sa partie aval. Vukovar en port danubien martyre regarde inquiet la Voïvodine. Le paradis naturel deltaïque fait office de nouveau lieu de focalisation des dissensions entre Roumains et Ukrainiens en laissant toujours peu de place à la République de Moldavie. Le Danube balkanique retarde les efforts d'harmonisation et d'intégration continentale. Le cas du canal de Bystroe, nouveau dossier d'affrontement entre Bucarest et Kiev depuis l'été 2004 est exemplaire des tensions s'appuyant sur le fleuve et les difficultés de négociation interétatique dans cet espace.

5 Lien voulu ou non, marginal ou stratégique, «le roi des fleuves de l'Europe » selon l'expression de Bonaparte, occupe une place singulière sur le continent. Mais jusqu'à quel point peut-on considérer qu'il influence la coopération des pays de son bassin ? Sa partie balkanique souffre d'une histoire récente déstabilisée. Dans une Europe en recherche de repères, le Danube peut-il susciter une mobilisation constructive, un support potentiel de solidarité?

\section{Les éléments d'internationalité du Danube}

Le bassin du Danube constitue une zone de contacts intenses. La compréhension de ses éléments constitutifs est essentielle pour percevoir les enjeux géopolitiques de l'élargissement de l'Union européenne. Plus grand fleuve d'Europe après la Volga, le Danube est un fleuve transcontinental qui s'écoule sur $2850 \mathrm{~km}$ de la Forêt noire à la mer Noire. Il fait l'objet d'une segmentation politique telle, qu'il est de loin le fleuve le plus international au monde.

\section{Le Danube, un fleuve segmenté en trois sous-ensembles}

7 Le relief, le climat et le régime hydrologique du fleuve conditionnent en partie le peuplement et l'organisation historique des ensembles politiques du bassin danubien et de l'Europe centrale et orientale. Le Danube présente un cadre morcelé. La morphologie 
du continent favorise l'émergence de micro-univers relativement séparés. C'est pourquoi on change presque autant de langues que de pays le long du fleuve.

Plus que des unités, la géographie physique définit certaines convergences. Ainsi l'interdépendance des espaces alpins, sudètes et carpatiques invitent selon l'historien François Fejtö ${ }^{3}$ à une union politique comme l'empire austro-hongrois. Cette tendance au rapprochement des peuples vers les plaines du bassin constitue une des clés de la cohésion stratégique entre l'Autriche et la Hongrie sous l'Empire. Michel Foucher le montre bien à travers son étude des frontières ${ }^{4}$ en Europe centrale et orientale.

9 Ainsi on retrouverait de grandes distinctions politiques influencées par le relief : les régions alpines montagneuses formant le Danube hercynien sont germaniques; les plaines pannoniennes cernées de chaînes de montagnes ont favorisé l'installation magyare et le rapprochement de l'Autriche et de la Hongrie ainsi que leur domination sur les Slovaques et les Croates. Enfin, la partie sud de la chaîne subcarpatique et les gorges des Portes de Fer coupent l'extrême cercle de l'occupation ottomane d'une région de plaines, roumaines au nord et bulgares au sud, pour lesquelles le Danube a offert une certaine possibilité de matérialiser les frontières (figure 1).

10 Toutefois, le degré explicatif de l'organisation géopolitique autour de l'axe fluvial par les caractéristiques de l'environnement et du milieu géomorphologique danubien n'est évidemment que partiel. L'idée selon laquelle il existe des frontières naturelles n'est pas fondée. Néanmoins, le Danube pose de réelles limites physiques. La contrainte danubienne est multiple et s'accroît vers l'aval. Cet obstacle trace une cicatrice profonde entre des régions au développement inégal. De plus, ses inondations fréquentes et importantes n'ont pas favorisé l'installation humaine sur ses rives.

11 La constitution d'ensembles politiques s'appuie aussi sur des dynamiques sociales d'initiatives, de réactions et de choix endogènes ou conditionnés par l'extérieur, largement transmises par héritage. On retrouve sur le Danube les grandes lignes de partage du continent européen et les stigmates d'une histoire mouvementée.

\section{L'histoire du Danube, un balancement permanent entre unité et différenciation}

figure 1 : Représentation schématique du peuplement du bassin du Danube : 


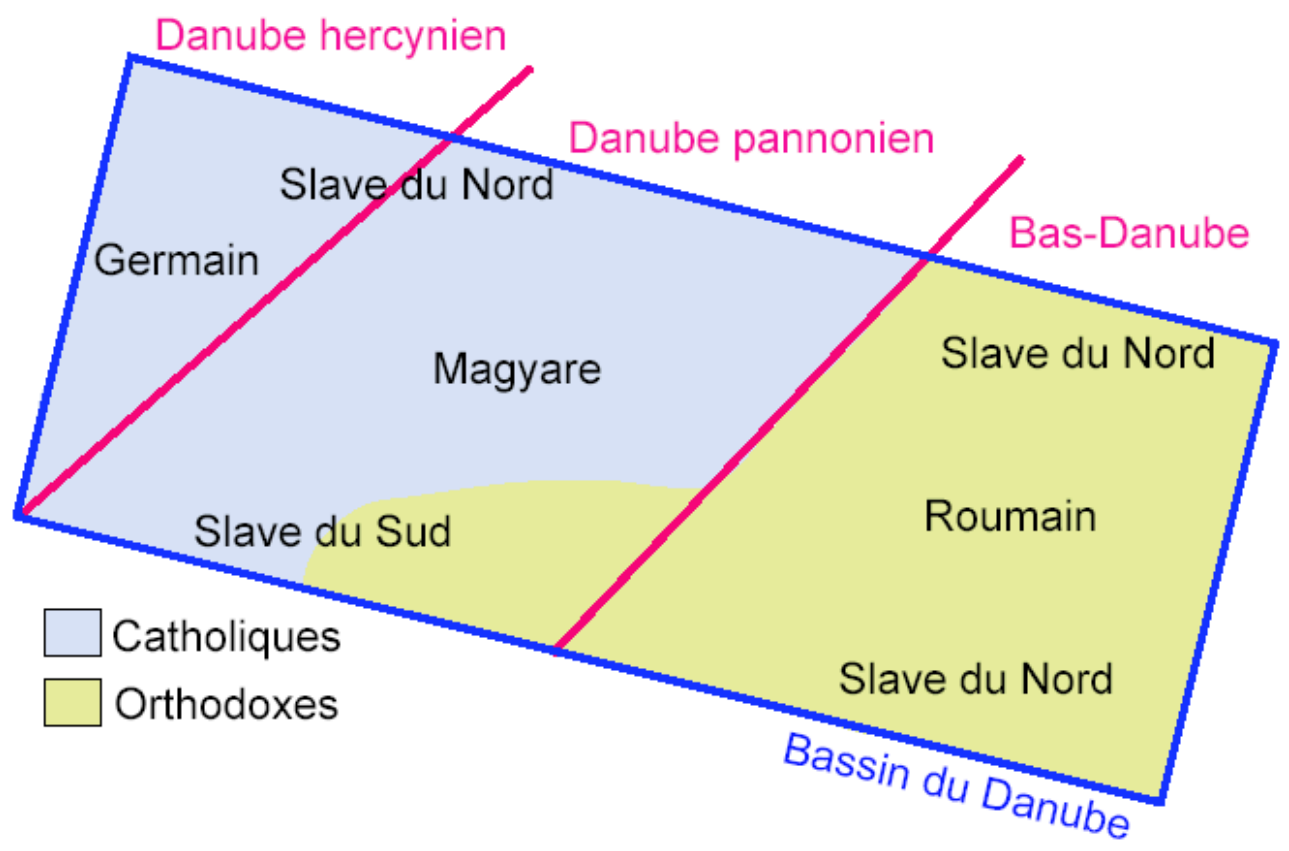

Source : élaboration personnelle ${ }^{5}$. prédominant. La domination ottomane accentue les contrastes entre la partie occidentale, solidement intégrée à l'Europe de l'ouest qui allait participer à son essor économique et la partie orientale du bassin désormais incorporée à l'Empire ottoman. Celle-ci se caractérise par un immobilisme croissant à l'écart des nouveaux circuits commerciaux atlantiques. L'ouverture des voies maritimes vers l'Asie et l'extrême 
orient finit de rendre désuète la voie danubienne en même temps que l'Empire ottoman contourné rentre en crise.

Devenu l'enjeu de la rivalité des grandes puissances, la partie aval du fleuve se trouve après 1880 partagée entre petits États qu'opposent des rivalités territoriales, sur fond d'hégémonies russe et autrichienne. La monarchie des Habsbourg développe la navigation sur le fleuve avec la célèbre Donau-Dampfschifffahrtsgesellschaft (D.D.S.G.), compagnie constituée en 1829 avec participation anglaise. Avec les progrès techniques, l'ère des grands aménagements assure le développement des berges du Danube.

$\mathrm{Au} \mathrm{XX}^{\mathrm{ème}}$ siècle, la destruction des empires austro-hongrois et ottoman laisse place à une mosaïque d'États sur le Danube. L'unité du réseau navigable est rompue. Finalement, la restructuration nationale débouche sur des économies plus concurrentes que véritablement partenaires. La Première guerre mondiale marque la fin de l'ancienne solidarité entre régions industrielles et agricoles. Le fleuve devient le lieu de partage entre la Petite Entente sous influence française et les États vaincus qui s'allient à l'Allemagne. Après 1945, le Rideau de fer achève de séparer la partie hercynienne du reste du fleuve, aire de domination de l'URSS.

Depuis 1989, le mouvement séculaire de fractionnement s'est poursuivi avec la séparation à l'amiable de la Slovaquie (1993) et l'implosion de la Yougoslavie. Dans le même temps, l'intégration régionale sous la houlette de l'UE s'affirme comme une composante incontournable des relations internationales modifiant la fonction des frontières dans l'Europe danubienne. Neuf ans après l'Autriche, dix pays de l'Est sont devenus membres de l'UE entre 2004 et 2007. L'entrée de la Roumanie et de la Bulgarie voire l'adhésion prochaine de la Croatie conforte la vocation européenne du Danube, en parallèle de la réorientation des flux vers l'Ouest.

La figure 2 présente la succession des régimes politiques, leur dimension spatiotemporelle ainsi que leur emplacement sur le cours du Danube (exprimé en $\mathrm{km}$ ). On voit parfaitement apparaître des fractures fortement inscrites dans la durée. Elles correspondent sensiblement aux trois sous-systèmes identifiés précédemment. 
Figure 2 : Présence historique des ensembles politiques sur le Danube : un espace fragmenté et régionalisé

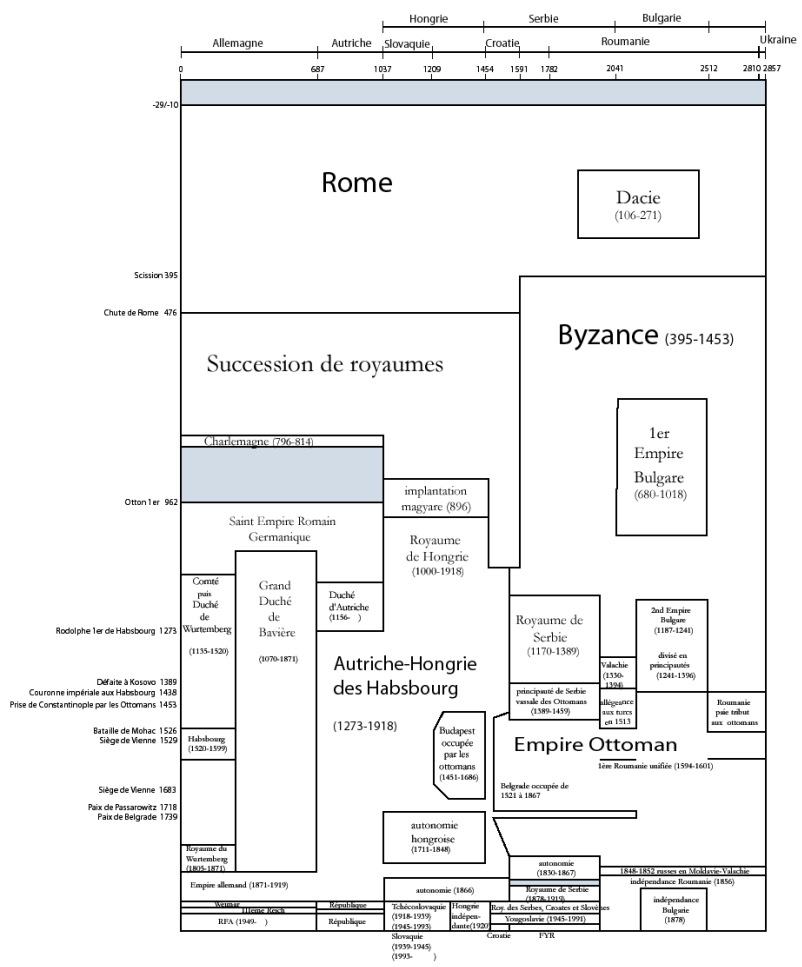

21 (NB : la possession du delta du Danube, hautement stratégique, n'est pas pris en compte ici)

\section{Les mythes de l'unité danubienne}

La structure et l'orientation des échanges jouent un rôle majeur mais ce sont aussi des conceptions géopolitiques qui décident du devenir des axes potentiels de communication. La volonté politique est indéniablement le facteur clé de l'organisation et de l'aménagement des territoires. A ce titre les projets prenant pour cadre un espace danubien rassemblé semblent aussi anciens qu'inexploités. On dispose de projets d'unions ou de fédérations danubiennes aussi abondants que différents dans leurs motivations et dans leurs objectifs et dont le nombre s'accroît alors que l'élargissement de l'UE apporte un cadre de réflexion renouvelée sur la région. Ces projets s'ancrent essentiellement vers l'Ouest et la sphère d'influence germanique. C'est pourquoi nous verrons successivement les projets et idées de coopérations et d'unions relevant d'une part de la question de l'identité autrichienne par rapport au modèle allemand que l'on présentera ensuite. 


\section{Le mythe habsbourgeois ou la croyance en une unité bénéfique pour tous} plusieurs aspects principaux. Il invite à la redécouverte des liens culturels et historiques qui unissent les citoyens d'Europe centrale et ceux de l'ouest. Il incite à repenser la place de l'Allemagne comme un pivot géographique au centre de l'Europe, débarrassé de prétentions impérialistes. Cette Mitteleuropa incarne des valeurs de paix et de détente. L'Allemagne a maintenant réglé ses derniers contentieux avec ses voisins et d'autres enjeux retiennent son attention. D'autre part, dans les pays d'Europe centrale et orientale, on peut estimer que «le principal souci n'est pas la bonne entente avec l'Allemagne mais leur intégration progressive dans le monde occidental $»^{8}$.

On parle de la monarchie danubienne (Donaumonarchie) pour dénommer les possessions de l'Autriche-Hongrie des Habsbourg. Cette terminologie ne reflète pas seulement des considérations géographiques. Tout un courant de pensée considère que cette période et son influence sur le bassin du Danube ont profondément marqué les peuples et l'organisation géopolitique européenne au point d'en faire un élément nécessaire à la stabilité du continent qu'il faudrait reconstituer.

habsbourgeois ${ }^{6}$ demeure intéressant pour comprendre les sentiments identitaires des peuples du bassin, leur intérêt pour une coopération et sa forme. L'empire d'AutricheHongrie a en effet dominé le bassin danubien pendant près de quatre siècles fondant son expansion sur la vallée du Danube suivant le refoulement des Ottomans. L'Europe centrale et orientale porte la marque de cette domination des Habsbourg.

points de vues, très antagonistes sur la nature de l'Empire, révèlent la recherche d'appartenance des pays et des peuples du bassin danubien. De ce mythe découle " l'effet Habsbourg ", la " reconstitution de solidarités économiques » et une volonté d'« amarrage à l'Occident $»^{7}$.

défensif contre les menaces extérieures. Le mythe habsbourgeois repose donc sur une ambiguité par rapport à un État dominateur et sur le choix de peuples qui l'auraient pourtant préféré à un autre. Comme la recherche de la Pax romana idéalisée après la chute de l'Empire romain « l'effet Habsbourg » est apparu dans une période de troubles faisant suite à une autorité dont les conditions de disparition sont équivoques.

\section{Les autres utopies ou la diversité des projets pour un espace convoité}

Le Danube est un axe de culture ambivalent et incomplet. Comparée à celle du Rhin, son imagerie apparaît bien plus diffuse, ne pouvant être mobilisée en totalité par aucun des États riverains. De manière générale, la relative faiblesse du Danube dans les panthéons mythiques, dansles constructions symboliques, les représentations artistiques (peinture, sculpture, littérature, musique...) contraste avec son influence physique sur la région. Il existe bien certains traits communs faisant penser à une culture danubienne, mais à bien des égards celle-ci reste une attache germanique. 
29 L'Europe danubienne peut paraître suffisamment diverse et hétérogène pour que son union politique reste une utopie intellectuelle masquant parfois des intérêts nationaux exacerbés. Le positionnement des Balkans entre Orient et Occident pose la question de la fonction de cette artère fluviale, trait d'union ou axe de rupture dans l'espace et le temps.

\section{Les intérêts pour l'unité du bassin danubien}

30 La coopération se base le plus souvent sur des éléments concrets, le symbolisme ne venant bien souvent qu'appuyer une intention politique. Le Danube, support de domination, a fait l'objet d'ambitions hégémoniques diverses. Il fournit en effet une attache territoriale à un grand nombre de pays. De plus, ses dimensions imposent de disposer de moyens financiers et techniques pour l'aménager et tirer bénéfice de sa présence.

\section{Un rôle économique contrasté : aménagements et recherche de cohérence globale}

31 La nature même du fleuve permet de discerner un intérêt géographique commun dans la maîtrise des crues. L'ampleur des problèmes à résoudre implique une intervention concertée au moins compte tenu des conséquences en aval des aménagements réalisés. Jusqu'au début de la révolution industrielle, les aménagements resteront essentiellement restreints à des constructions militaires. Les investissements requis, considérables, joints aux vicissitudes historiques ont longtemps empêché l'aménagement d'un espace danubien cohérent.

On distingue plusieurs étapes dans l'aménagement des fleuves européens. L'amélioration de la navigation va de paire avec les mesures pour limiter l'imprévisibilité du fleuve. Elle peut concerner directement les berges du fleuve ou bien la réalisation de canaux dont les plus célèbres ouvrent le Danube sur le Rhin via le Main (inauguré en 1992) ou sur la mer Noire (1984). Dans la deuxième génération de travaux, on trouve les barrages pour la production d'électricité, la régulation du débit, l'amélioration de la navigation, la constitution de réserves consommables, éventuellement l'irrigation. On compte près de 10000 barrages dans le bassin du Danube, concentrés dans sa partie alpine plus propice à ce type d'installation. L'intérêt d'actions conjointes apparaît alors indéniable. Cependant il n'est pas toujours évident de discerner la finalité stricte d'un projet dans cette zone de confrontations. Si le barrage des Portes de Fer constitue un exemple d'entente réussie, on ne peut en dire autant de celui de Gabcikovo-Nagymaros, véritable catalyseur des antagonismes hungaro-slovaques.

Déjà Adam Smith (1776) soulignait l'intérêt de la navigation dans le développement du commerce, dans l'extension des marchés et ainsi de la division internationale du travail. Il indiquait ${ }^{10}$ que le séquençage du Danube par de multiples frontières explique sa faible utilisation et que son unité profiterait à tous. Le Danube peut s'avérer une ouverture majeure pour des pays enclavés. Pour cela, il doit trouver sa place dans la restructuration des réseaux de transports européens. 

Noire et la mer du Nord. Selon Olivier Podevins, consultant en logistique, "l'axe Rotterdam-Mer Noire via le Rhin, le Main et le Danube se révèle de plus en plus structurant pour la Hongrie, la Slovaquie, la Slovénie et les pays balkaniques ${ }^{16}$. En réalité, les régions entre Vienne et Budapest captent l'essentiel des investissements. Dans les secteurs automobile et électronique, de nombreuses plateformes logistiques sont développées. Compte tenu des spécialisations industrielles, la navigation fluviale n'émerge cependant pas comme le mode de transport le mieux adapté. Mais plus en aval, le Danube traverse des régions très agricoles ou encore productrices de matières premières, intrants pondéreux susceptibles quant à eux d'un transport fluvial. 

de la Caspienne dont le transit oppose la Roumanie et la Bulgarie. L'intérêt pour la voie fluviale danubienne varie beaucoup d'un pays à l'autre. L'Autriche et l'Allemagne, principaux investisseurs à l'Est, se montrent très motivées par le trafic fluvial. Avec les élargissements le long du fleuve, ces pays retrouvent un espace privilégié de leur hinterland. La Roumanie place également beaucoup d'attentes dans le fleuve. Les autres pays se contentent plutôt de suivre le mouvement.

\section{Un patrimoine écologique exceptionnel à défendre}

La protection de l'environnement est un atout de la navigation fluviale comparée à la route qui constitue également un thème fédérateur pour tous les pays. Le fleuve représente le cas type de la manifestation des externalités. Celles-ci lui confèrent des caractéristiques singulières faites d'interdépendances et de responsabilités, de l'amont vers l'aval. Les implications d'être riverain du Danube rendent donc nécessaire une gestion internationale minimale. La pollution ne connaît pas les frontières et oblige à adopter une vision globale basée sur des activités localisables. L'environnement occupe ainsi une place centrale comme élément de coopération par son côté transversal et mobilisateur. Les aménagements sur le fleuve mettent actuellement l'accent sur la régulation des ressources en eau potable.

41 Le Danube peut apparaître comme une source de services et de croissance potentielle. Toutefois, il faut procéder à un arbitrage entre ce qui est concevable et les impacts sur un milieu écologique unique en Europe. Jusqu'au début des années 1990, dans des pays soumis à des régimes politiques centralisés et autoritaires, il est bien connu que les politiques menées n'avaient pas de sensibilité environnementale marquées et qu'il en a résulté entre autre un niveau de pollution des eaux élevé. Actuellement, l'UE accorde une place importante à la dimension environnementale dans les projets de développement ${ }^{17}$. Dans les faits, le Danube s'avère aujourd'hui un fleuve encore relativement préservé et finalement assez « libre » comparé bien sûr à ses homologues européens.

\section{La diversité des formes de coopération sur le Danube}

Le Danube lie ses riverains mais dissocie l'espace. Une organisation politique originale sur différents niveaux est née de la confrontation entre ces éléments. De natures et de formes très hétérogènes, ces entités posent la question de la cohérence des dynamiques de modifications des cadres territoriaux d'intervention politique. Dans le contexte actuel, les structures de concertation politique se multiplient dans l'Europe danubienne sous des formes très différentes selon leur degré d'institutionnalisation. Les mouvements de concertation récents laissent présager une intensification et une efficacité accrue de la coopération prenant le Danube comme fondement.

\section{Coopération ou domination?}

Suite à des guerres incessantes sur les parties centrale et inférieure du fleuve et le déclin du bassin danubien, le renouveau du fleuve constituera l'enjeu de la politique 
des grandes puissances des XVIII ${ }^{\text {ème }}$ et XIX ${ }^{\text {ème }}$ siècles, en particulier avec l'établissement de son statut et sa segmentation entre ses parties maritime et continentale.

Les divergences d'intérêts entre les puissances européennes ont rendu difficile la définition d'un statut du Danube bien que celui-ci ait été proclamé fleuve international par le traité de Vienne (1815). L'Autriche-Hongrie cherche à contrôler le commerce des céréales roumaines sur le Danube et en mer Noire et s'est assuré la liberté de navigation en amont par le traité signé en 1851 avec la Bavière et le Wurtemberg. Les Habsbourg sont favorables à une internationalisation du fleuve en territoire ottoman mais refuse toute intervention étrangère sur leur territoire. La Grande-Bretagne est surtout attachée au libre accès des embouchures. La Russie qui subit la concurrence des céréales danubiennes sur sa production ukrainienne s'intéresse plutôt au commerce occidental que danubien.

La nécessité d'un compromis a empêché la mise en place d'un statut couvrant l'ensemble du Danube. Le traité de Paris (1856) signé sous l'influence de la victoire franco-britannique en Crimée, a proclamé la liberté de navigation pour tous les États et interdit toute perception de taxe au profit des États riverains. Il a soumis le secteur maritime à la juridiction d'une Commission européenne aux privilèges d'extraterritorialité étendus et composée des représentants des grandes puissances et de la Turquie. L'autre instance internationale prévue pour l'ensemble du cours et où devaient siéger les États riverains n'a jamais été constituée en raison de l'opposition de l'Autriche-Hongrie et de la Roumanie, remplaçant la Turquie en vertu du traité de Berlin (1878). Par ce traité, l'Autriche-Hongrie se faisait attribuer la responsabilité de l'aménagement continental, limitant ainsi efficacement le ressort des institutions internationales où s'exerçait l'influence britannique. Appuyée par l'Allemagne, elle utilisait alors le Danube comme un instrument de sa politique d'expansion vers l'Est. A l'embouchure, la Russie était soumise à un régime spécial limitant les aménagements susceptibles de nuire à la navigabilité des autres bras du delta.

Jusqu'en 1914, le Danube comportait ainsi sur le plan politique et économique deux secteurs, l'un prolongement des routes maritimes et soumis aux règles du commerce maritime international, l'autre continental sous le contrôle des puissances d'Europe centrale.

Pour favoriser les aménagements et faire appliquer les traités de 1919-1920, les Alliés utilisèrent différents moyens dont la création de commissions de navigations sur le Rhin, le Danube, l'Elbe, l'Oder. Avec le traité de Paris (1921), la coopération sur le Danube atteignait un point maximum d'internationalisation. Tout le cours du Danube est confié à une Commission internationale formée des États riverains, de la France, de la Grande-Bretagne et de l'Italie. Cette commission exerce un pouvoir d'administration et de surveillance. L'ancienne Commission européenne continuait à gérer le secteur maritime, soustrait à la souveraineté de la Roumanie.

La crise économique de 1929 va laisser de profondes traces. Dans cet espace économique disloqué, les tendances autarciques et protectionnistes vont se développer. On assiste à la destruction de la coopération via l'action des nazis dans les institutions internationales danubiennes scellant leur disparition progressive. Le domaine de gestion de la Commission va se réduire au fur et à mesure des annexions hitlériennes. La Commission qui ne peut plus fonctionner est alors remplacée par une commission provisoire créée par le Reich qui y admet l'URSS. La Commission européenne qui gérait l'embouchure (France, Grande-Bretagne, Italie, Roumanie) accepte ensuite la 
souveraineté roumaine sur tout le cours inférieur en vertu de l'accord de Sinaïa (1938). Sulina perdait son statut de port international. Le traité de Bucarest (1939) voit l'entrée du III Reich dans cette Commission qui termina ses activités en 1940.

La Convention de Belgrade (18 août 1948) ne permet qu'aux seuls pays danubiens de participer à une nouvelle et unique Commission du Danube mais garantit la libre circulation sur le fleuve. Suivant la volonté de Staline, la Convention réaffirme la souveraineté des États riverains qui disposent par exemple de la capacité d'autoriser ou non le cabotage et reçoivent l'obligation d'entretenir les berges. Après la Seconde guerre mondiale, l'axe danubien devient un instrument de domination de l'URSS dans le Conseil d'Assistance Economique Mutuel (CAEM). La rupture entre Tito et Staline (déclaration du Kominform du 28 juin 1948) n'a en la matière pas empêché une convergence soviéto-yougoslave de type souverainiste pour exclure les États non riverains des affaires danubiennes. Les sanctions économiques contre la Yougoslavie limiteront toutefois le développement des échanges jusqu'à la déstalinisation.

Les traités successifs, une volonté de coopérer émerge et s'impose tardivement

1616 : Traité austro-turc signé à Belgrade par lequel les Autrichiens bénéficient du droit de naviguer sur le milieu et sur la partie basse du fleuve ;

1774 : Traité de Küçük Kaynarca, permet à la Russie d'utiliser le bas-Danube ;

1829: Traité d'Andrinople, ouvre les Détroits (fin du monopole ottoman sur la navigation en mer Noire)

1840: Convention de Saint-Pétersbourg russo-autrichienne, liberté de naviguer sur l'ensemble du Danube ;

1851 : Traité entre l'Autriche-Hongrie, la Bavière et le Wurtemberg visant à assurer la liberté de navigation ;

1856 : Traité de Paris, précise le principe d'internationalisation du fleuve et établit la première Commission du Danube ;

878: Traité de Berlin, rétablit l'équilibre européen aux dépens de la Russie; la Roumanie indépendante remplace la Turquie à la Commission ; l'Autriche-Hongrie se fait attribuer la responsabilité de l'aménagement du secteur des cataractes; le bas Danube est déclaré zone neutre ;

1921 :Traité de Paris, pose le statut du Danube admis par l'Autriche, l'Allemagne, la Yougoslavie, la Bulgarie, la Roumanie, la Grande Bretagne, l'Italie, la Belgique, la Tchécoslovaquie, la Hongrie et la Grèce ; la Convention du 23 juillet 1921 confie tout le cours du Danube à une Commission internationale (institution officielle aux pouvoirs larges, ayant son propre drapeau, le droit de prélever des taxes, l'immunité diplomatique de ses membres); l'ancienne Commission des États riverains est rebaptisée Commission internationale du Danube ; 1938 : Accord de Sinaïa, la Roumanie retrouve sa souveraineté sur la partie maritime ; 1939 : Traité de Bucarest, entrée du Reich dans la Commission européenne ;

Un accord signé le 12 septembre à Vienne abolit la Commission internationale du Danube la remplaçant par un Conseil du Danube fluvial (excluant la France et la Grande-Bretagne) ; 
1945 : Postdam (juillet), le président Truman propose qu'un accord international rétablît la libre navigation sur le Danube. L'URSS refusa d'inscrire la question du Danube dans les traités de paix puis finit par accepter un article décidant que «la navigation sur le Danube serait libre et ouverte pour les nationaux, vaisseaux de commerce et marchandises de tous les États, sur un pied d'égalité ». Le conseil des ministres des Affaires étrangères décida, le 6 décembre 1946, qu'une conférence sur le Danube serait convoquée six mois après la mise en application des traités de paix (qui entreront en vigueur le 15 septembre 1947).

1948 : Convention de Belgrade, reconstitue la Commission du Danube (seule l'Allemagne de l'ouest ne rejoint pas la convention (statut d'observateur) l'Autriche la rejoint seulement en 1960).

Après la chute des régimes socialistes, la situation géopolitique du Danube est particulièrement renouvelée. L'héritage communiste est un élément ambivalent dans la mesure où avant 1989, ces pays se percevaient moins comme partenaires que comme concurrents. La Yougoslavie se présentait comme socialiste mais revendiquait un statut extérieur au bloc communiste. La Roumanie appartenait au CAEM mais pas au Pacte de Varsovie. La Hongrie avait pris des réformes rapprochant son économie de celle d'un pays capitaliste. La coopération régionale n'intéressait pas ces pays qui cherchaient à attirer les investisseurs étrangers et à distinguer leurs systèmes politiques du système soviétique. Mais la sortie au même moment du bloc de l'Est et le traumatisme postcommuniste qui touche leurs sociétés les rapprochent au moment où se recomposent les alliances.

\section{Les institutions internationales : le Danube comme point d'ancrage}

7 Les relations internationales danubiennes relèvent essentiellement de considérations techniques. Elles ne se veulent pas le support de valeurs et d'une coopération politique approfondie. Le Danube ne serait-il que le support de préoccupations sectorielles?

La Commission du Danube ${ }^{18}$ compte les onze pays riverains comme membres (les PaysBas et la France sont observateurs) et siège à Budapest. Fondée en droit international sur la Convention de Belgrade (1948) et en droit privé sur les Accords de Bratislava (1955), elle a pour mission principale d'assurer la libre navigation. Elle élabore également les projets de grands ouvrages et exécute dans certains cas les travaux. Elle contribue à l'harmonisation des systèmes de navigation et des formalités douanières et sanitaires et effectue un travail de documentation. Composée d'un représentant par membre, la Commission du Danube élit parmi eux son président, son vice-président et son secrétaire pour une période de 3 ans. La Commission du Danube dispose, pour s'acquitter de ses tâches, d'un secrétariat comprenant des conseillers spécialisés et d'un directeur général. Ses langues officielles sont l'allemand, le français et le russe.

Suite à la destruction du pont de Novi Sad en avril 1999 par l'OTAN, bloquant ainsi la navigation sur le Danube, la Commission du Danube a montré, dans sa manière de surmonter la crise, une certaine efficacité bien qu'on l'ait beaucoup sous-estimée. Elle souffre néanmoins d'un handicap structurel lié au caractère non contraignant de ces décisions. L'organisation de ses travaux revêt toujours un formalisme pesant qui paraît hérité du passé. L'Allemagne et l'Autriche ont manifesté une volonté claire de réformer en profondeur ses méthodes de travail. Le processus de préparation pour la révision de la Convention de Belgrade a commencé en 2003 avec deux groupes de travail, l'un sur la 
navigation coordonnée par la Roumanie, l'autre sur l'aspect institutionnel et juridique par l'Autriche. Le Comité préparatoire doit élaborer des amendements et/ou additions à la Convention de Belgrade à l'intention d'une conférence diplomatique des États membres.

Depuis 1998, la Commission internationale pour la protection du Danube(ICPDR) ${ }^{19}$ réunit les pays du bassin pour préserver cet écosystème exceptionnel. Bien qu'assez ancienne dans le bassin du Danube, la concertation sur le thème de l'écologie s'est accrue et organisée après 1989 dans le cadre de programmes regroupant différents pays, les institutions internationales (Banque mondiale, agences des Nations unies, Commission européenne) et des ONG. Cette nouvelle commission dispose d'un groupe d'experts permanents et temporaires et organise régulièrement des forums pour réunir tous les intéressés. Malgré de bons résultats, cette organisation manque encore de moyens et de cohésion.

71 La création d'une nouvelle entité démontre le manque d'intérêt pour l'environnement de la Commission du Danube et son long blocage par les confrontations entre ses membres. Depuis janvier 2001, elle a cependant commencé la mise en œuvre d'un projet d'assainissement de certaines parties du Danube. Conscientes des interactions entre les milieux naturels, la Commission internationale pour la protection du Danube et la Commission de la mer Noire (ICPBS) ont signé un mémorandum à Bruxelles le 26 novembre 2001 afin de favoriser la concertation et leurs actions conjointes fondées sur des objectifs stratégiques communs. Une Task Force informelle baptisée DABLAS a été créée, présidée par la Commission européenne. Les objectifs en la matière sont déjà de rapprocher les interlocuteurs et d'inciter à l'harmonisation des normes et des pratiques.

72 La Commission du tourisme sur le Danube réunit depuis 1972 tous les pays riverains (à l'exception de la République de Moldavie et de l'Ukraine) dans une association dépassant les clivages idéologiques de l'époque. Elle a pour vocation d'aider à prendre des mesures homogènes et coordonnées pour favoriser l'essor touristique du fleuve et depuis 1998 du canal Main-Danube.

73 Le rapprochement vers les organisations de l'Ouest a été conduit en parallèle à la mise en œuvre de systèmes de coopération régionale entre les pays d'Europe de l'Est finissant d'achever l'ancien régime soviétique d'alliances. Une multitude d'initiatives régionales et transnationales a émergé dans l'Europe du Sud-Est. Ce nouvel espace de coopération est doté d'un réseau de relations de toutes natures, institutionnelles, économiques, politiques et commerciales. Ces structures sont d'une extrême variété dans leurs statuts, leurs fondements et leurs actions. On trouve pêle-mêle l'Initiative Centre Européenne (ICE), la Zone de Coopération Economique de la Mer Noire (ZCEMN), l'Initiative de coopération d'Europe du Sud-Est (ICESE), l'Accord de libre-échange centre européen (ALECE) chacune recouvrant plus ou moins le bassin danubien. Devant cette profusion, on peut s'interroger sur leur efficacité réelle.

74 Le Pacte de stabilité pour l'Europe du Sud-Est (PSESE) forme la principale structure de coopération dans cette partie du continent avec le plus grand nombre de participants. Forum politique créé le 10 juin 1999 à l'initiative de l'Allemagne suite au conflit au Kosovo, le Pacte intervient dans les questions liées à la démocratisation et aux droits de l'Homme, à la reconstruction économique et à la sécurité et la défense. Cette instance de coordination veut susciter des initiatives nouvelles via un raccordement à l'UE. 
75 La coopération régionale en Europe centrale et orientale s'appuie sur des structures souples et des arrangements pragmatiques tout en évitant les questions sensibles. Ces constructions régionales se présentent généralement comme un «surplus » institutionnel, éventuellement facile à démanteler. Le refus d'institutionnalisation du groupe de Visegrád est flagrant. Ce ne sont que des institutions transitoires, des structures diplomatiques préalables à une intégration dans l'UE. L'adhésion rendant caduque la poursuite d'efforts régionaux, la volonté politique a largement fait défaut pour un quelconque approfondissement. Cette recherche de crédibilité constitue certainement une motivation plus réelle de ces démarches que la perception d'un intérêt direct à collaborer.

76 Avec le Processus de coopération danubienne,le bassin du Danube dispose d'un cadre intergouvernemental. Il a été lancé le 27 mai 2002 à Vienne par une déclaration signée par les représentants des treize États danubiens, le commissaire aux relations extérieures de l'UE, Chris Patten et le coordinateur spécial du Pacte de stabilité pour l'Europe du Sud-Est, Erhard Busek. On y retrouve la Commission du Danube, l'ICPDR, l'ICE, le PCESE, l'Initiative adriatique et ionienne et le comité directeur du corridor VII. Ce processus fonctionne à travers une conférence annuelle entre hauts-fonctionnaires et une rencontre tous les deux ans au niveau ministériel.

77 Son objectif global est de " promouvoir une coopération diversifiée afin de créer un espace de prospérité et de progrès dans la région du Danube » et de "développer la compréhension et la solidarité entre les membres ainsi qu'à promouvoir l'identité et l'intégration européenne ${ }^{20}$. Ce processus multisectoriel vient en soutien aux différentes institutions internationales décrites précédemment. Des évènements culturels biannuels sont mis en place avec la Conférence danubienne sur l'Art et la Culture. Le potentiel inexploité du fleuve est mis en avant ainsi que l'intérêt à établir des eurorégions et différentes formes de coopération transfrontalière et interrégionale.

78 La participation de l'UE n'occulte cependant pas la question de son utilité réelle, étant donné son manque de structure et de cadre d'intervention. Sans secrétariat, sans moyens financiers ni budget, ce processus se borne à des déclarations d'intentions et à un rôle d'encouragement à l'intégration à l'UE.

79 Face à l'effervescence des initiatives conduites plus ou moins spontanément dans le bassin du Danube, un besoin de clarté et d'institutionnalisation se fait ressentir. Ce cadre doit gagner en efficacité. Pour la première fois l'unification du Danube de la source à l'embouchure est potentiellement réalisable. Tout dépend de la mobilisation des acteurs institutionnels pour faire jouer les complémentarités. Les gains de la coopération sur le Danube se feront ressentir à moyen ou long terme. La continuité du processus de coopération du Danube semble donc nécessaire, tout comme son évolution et sa réforme sont inévitables. On peut imaginer la coopération autour du Danube se dissoudre dans l'UE, mais c'est peu probable. Elle porte une dimension symbolique et des initiatives qui s'intègre à l'UE mais auxquelles celle-ci ne peut se substituer totalement.

80 À l'UE elle-même, il manque une vision. Son projet pour le fleuve se cantonne peu ou prou aux engagements actuels. Un projet d'avenir inclusif et structuré, tel est l'enjeu véritable du devenir de la coopération danubienne si elle ne veut pas se fondre et disparaître dans l'UE ou se borner au règlement de questions techniques et sectorielles. À travers la multitude d'initiatives locales, le bassin du Danube pourrait ouvrir la voie à un enrichissement institutionnel inédit et contribuer à améliorer la place des régions 
dans l'Europe. Cette importance géopolitique du Danube se conçoit pour le développement du bassin en lui-même mais aussi vis-à-vis de l'extérieur du côté de la mer Noire.

81 L'Europe doit faire face à de nouveau enjeux: mondialisation, respect de l'environnement et trafics en tout genre. Le Danube en est un laboratoire. Il occupe une place privilégiée pour la cohésion territoriale dans une UE à l'hétérogénéité croissante mais qui offre la seule alternative pour « que ce fleuve cesse d'être divisé par des frontières en empires, et qu'il devienne l'artère principale d'une Europe centrale unie, le médiateur pacifique de l'Orient et de l'Occident $»^{21}$.

\section{NOTES}

1. p. 332 in Magris (Claudio), Danube,Paris : L'Arpenteur, 1988, 441 p.

2. p. 228 in Béthemont (Jacques), Les grands fleuves. Entre nature et société, Paris : Armand Colin, 1999,255 p.

3. Fejtö (François), Requiem pour un empire défunt - Histoire de la destruction de l'Autriche-Hongrie, Paris : Points, 1992, 464 p.

4. Foucher (Michel), Fronts et frontières un tour du monde géopolitique, Paris : Fayard, 1991, 691 p. En particulier, la carte «L'Europe : un faisceau de frontières » (annexe de la table des cartes p.74) montre bien l'extraordinaire mouvance des tracés sur la partie centrale du fleuve et la grande stabilité à l'amont.

5. NB : Slave du Nord: installation vers le V ème siècle environ (Pologne, Tchéquie, Slovaquie, Slovénie, Bulgarie) ; Slave du Sud : aux alentours du IX ${ }^{\text {ème }}$ siècle (Croatie, Serbie).

6. Thèse de doctorat de Magris (Claudio), Le Mythe de l'Empire, Paris : Gallimard, 1991

7. Wild (Gérard), À propos de "l'effet Habsbourg ", Paris: Centre d'études prospectives et d'informations internationales (CEPII), mai 1987, 6p. (Note non publiée)

8. Rey (Violette) (sous la dir.), Les territoires centre-européens. Dilemmes et défis. L'Europe médiane en question, Paris : La Découverte, 1998, $264 \mathrm{p}$.

9. Cf. Marmorat (Marion), Controverse socio-technique autour des barrages danubiens GabcíkovoNagymaros, 1977-2004 (Hongrie/Slovaquie). Etude microsociologique des relations internationales, thèse de doctorat sous la direction de Bertrand Badie, Institut d'Etudes Politiques de Paris, 2006, 574 p.

10. p.28 in Smith (Adam), Inquiry into the nature and causes of the Wealth of nations, New York: Prometheus Books, 1991, 590 p.

11. Les données de la Commission du Danube montrent que le Danube était 3 fois moins utilisé que le Rhin en terme de quantités transportées en 2002 et qu'il est encore loin de son trafic record de 1986.

12. Cf. www.aivp.org

13. Cf. p.47 Commission européenne, Livre blanc. La politique européenne des transports à l'horizon 2010 : l'heure des choix, Luxembourg: Office des publications officielles des Communautés européennes, 2001, 128 p.

14. Les Corridors paneuropéens de transport établis à Helsinki (juin 1997) donnent une place privilégiée au Danube, Corridor ${ }^{\circ} \mathrm{VII}$ (www.corridor7.org).

15. Schwetz (Otto), The Danube - There is good news, BlackSeaTrans, n³, 2004, pp. 11-12 
16. Minefi DGTPE, Revue Elargissement Spécial Logistique, Dossier 51, Février 2005, 4 p.

17. Commission européenne, Renforcer les normes environnementales à travers l'Europe, Luxembourg : Magazine de la Direction générale de l'environnement $n^{\circ} 9$ février 2002, $16 \mathrm{p}$

18. Cf. www.danubecom-intern.org

19. Cf. www.icpdr.org

20. Déclaration finale de la Seconde conférence intergouvernementale du processus de coopération du Danube, Bucarest, 14 juillet 2004

21. Zweig (Stefan), «Un voyage sur le Danube il y a deux cents ans », in Voyages, Paris : Belfond, 2000, article publié à l'origine dans la revue Donauland, Vienne, mars 1917, p. 149.

\section{AUTEUR}

\section{JEAN-MARIE GAUTHEY}

Chargé de projets dans les infrastructures énergétiques en Europe centrale, auteur de « La recomposition géopolitique de l'espace danubien : entre permanences, reconstructions et intégration », mémoire de DEA sous la direction de M. Laurent Carroué, Institut d'Etudes Européennes, Université Paris 8, 2004, 301p. 